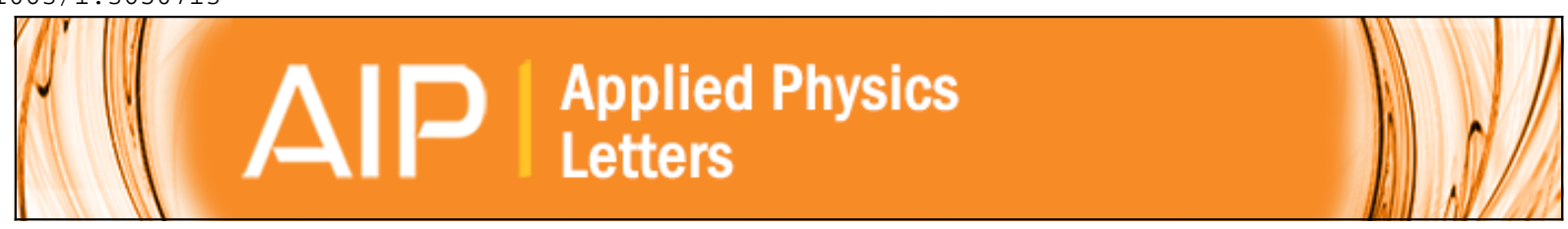

\title{
Enhanced dc magnetic field sensitivity by improved flux concentration in
} magnetoelectric laminates

Junqi Gao, David Gray, Ying Shen, Jiefang Li, and D. Viehland

Citation: Applied Physics Letters 99, 153502 (2011); doi: 10.1063/1.3650713

View online: http://dx.doi.org/10.1063/1.3650713

View Table of Contents: http://scitation.aip.org/content/aip/journal/apl/99/15?ver=pdfcov

Published by the AIP Publishing

AlP Re-register for Table of Content Alerts 


\title{
Enhanced dc magnetic field sensitivity by improved flux concentration in magnetoelectric laminates
}

\author{
Junqi Gao, ${ }^{\text {a) }}$ David Gray, Ying Shen, Jiefang Li, and D. Viehland \\ Department of Materials Science and Engineering, Virginia Tech, Blacksburg, Virginia 24061, USA
}

(Received 6 July 2011; accepted 20 September 2011; published online 10 October 2011)

\begin{abstract}
In this letter, we present magnetostatic modeling results that show significant magnetic field concentration tunability through geometric modification of high-mu magnetostatic Metglas layers of laminate magnetoelectric (ME) sensors. Based on the modeling results, composite ME sensors were fabricated with longer Metglas foils and found to exhibit notably higher ME voltage coefficients at smaller DC magnetic biases in response to a $1 \mathrm{kHz}$ driving signal. Such ME sensors have been used to detect DC magnetic field changes as small as $6 \mathrm{nT}$ at $1 \mathrm{kHz}$, while maintaining a signal-to-noise ratio greater than 10 . This represents an enhancement of $\sim 250 \%$ relative to values previously reported for Metglas/ $\mathrm{Pb}(\mathrm{Zr}, \mathrm{Ti}) \mathrm{O}_{3}$ laminates. (C) 2011 American Institute of Physics. [doi:10.1063/1.3650713]
\end{abstract}

Certain migratory animals are believed to have the capability of long distance navigation by detecting variations in Earth's magnetic field. ${ }^{1,2}$ The intensity of Earth's field ranges roughly between 0.25 Oe to $0.65 \mathrm{Oe}$, with different declination and inclination angles over the globe. The National Geophysical Data Center has developed very accurate models from which database cataloging geomagnetic field strength and direction have been generated. ${ }^{3}$ Coordinated use of this database and orthogonal vector magnetometers could offer for a global positioning system (GPS). The average of Earth's magnetic field gradient in the United States is around $0.02 \mathrm{nT} / \mathrm{m}$; thus, DC magnetic sensors with a sensitivity of several nanoTesla could detect the magnetic field variations over lengths of $100 \mathrm{~m}$, potentially enabling navigation.

Practical use of such a magnetic GPS would require the availability of highly sensitive DC magnetic sensors. To date, the most sensitive magnetic sensors are superconducting quantum interference devices (SQUIDs). ${ }^{4} \mathrm{~A}$ more economical, albeit less sensitive, sensor type is the class of sensors based on the giant magnetoresistance (GMR) effect. ${ }^{5}$ Existing conventional magnetic sensors, however, have several potential limitations for practical use. SQUIDs, for example, require extremely low operational temperatures, while GMR effects require considerable operational power.

The magnetoelectric (ME) effect offers an alternative type of magnetic sensor. ${ }^{6}$ Multi-phase laminated ME composites have been found, which have relatively giant ME voltage coefficients. ${ }^{7}$ Long, sandwich-type laminate structures composed of magnetostrictive Metglas and piezoelectric $\mathrm{Pb}(\mathrm{Zr}, \mathrm{Ti}) \mathrm{O}_{3}$ (or PZT) layers have values of up to $22 \mathrm{~V} / \mathrm{cm}$ Oe. ${ }^{8}$ Recently, DC magnetic sensors based on Metglas/PZTfiber laminates have been shown capable of sensing the intensity and inclination of Earth's field. ${ }^{3}$

Here, we have developed a magnetostatic finite element model to study the effects of in-plane magnetostrictive phase geometry on the magnetic flux concentration within high-mu

\footnotetext{
${ }^{\text {a) }}$ Author to whom correspondence should be addressed. Electronic mail: junqi08@vt.edu.
}

layers. Based on the predictions, we have redesigned the Metglas/PZT composites to incorporate longer Metglas foils. Increasing the axial dimension of the sensor results in an increase in the ME voltage coefficient under small DC magnetic bias fields in the range of $-2.5 \mathrm{Oe}<H_{d c}<2.5 \mathrm{Oe}$. This enhancement in the value of slope results in a 2.5 times increase in the DC magnetic field sensitivity when the laminate is driven at $1 \mathrm{kHz}$.

Magnetostatic modeling was performed using a commercial finite element modeling (FEM) package (Ansoft's Maxwell 3D). A uniform DC magnetic field was simulated by using a pair of neodymium permanent magnets separated by $25 \mathrm{~cm}$ at either end of the axial direction of the Metglas ribbons, as illustrated in Fig. 1(a). The strength of the neodymium magnets was adjusted to provide a sufficiently small $\mathrm{H}$ field, so as not to reach saturation $\left(\mathrm{H}_{\mathrm{sat}}\right)$ within the high mu material. A $1 \mathrm{~cm}$ wide by $25 \mu \mathrm{m}$ thick ribbon of high-mu material was placed between the bias magnets, and then the length was changed for various laminates between 80 and $100 \mathrm{~mm}$. The mu-metal was assigned a non-linear B-H curve (see inset in Fig. 1) from the Ansoft materials library file to approximate the real behavior of the Metglas. An automatic 1000 point mesh was generated within a control volume with appropriate boundary conditions, located sufficiently far from regions of interest within the material. The simulation was completed to within $0.5 \%$ accuracy after 100 iterations of the code.

The magnetic flux in the space close to the Metglas was found to be dramatically influenced by the high permeability of the foils. A planar representation of the $\mathrm{H}$ field in the space surrounding the Metglas is shown in Fig. 1(b). The magnetizing field (and corresponding flux density) was distorted in regions of close proximity to the Metglas in the Y-Z plane. The flux density was similarly distorted in all three dimensions surrounding the Metglas, although only a single plane is shown in Fig. 1(b) for illustration. The net effect of the flux concentration of the high-mu material was evidenced by a relative change in the internal field characteristics.

As shown in Fig. 2(a), the in-plane magnetic field strength for longer Metglas foils was larger than that of 

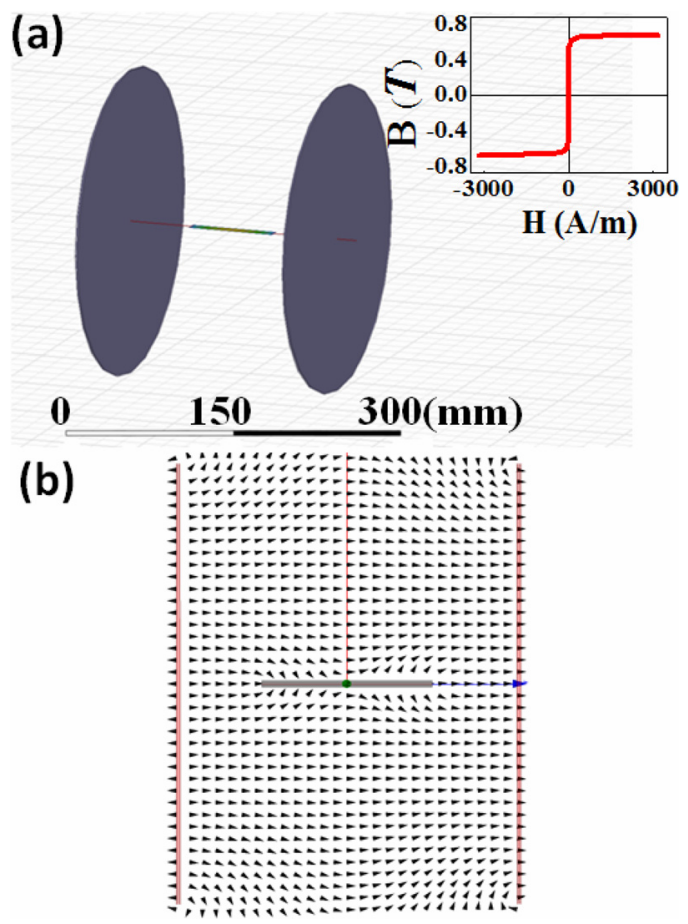

FIG. 1. (Color online) (a) Schematic representation of 3-D mangetostatic model layout including large, permanent magnetic $H_{d c}$ bias generators and (b) vector map of the $\mathrm{y}-\mathrm{z}$ (axial-height) component of the $\mathrm{H}$ field in the presence of the high-mu Metglas. Insert: non-ideal B-H relationship used to define magnetostatic behavior of high mu Metglas in FEM.

shorter ones, especially in the center portion of the foil. Line scans along the axial center-line of the Metglas foils (see Fig. 2(b)) with increasing length of the foil from 80 to 100 $\mathrm{mm}$ resulted in a $36 \%$ increase in field strength at the center of the Metglas ribbon. While the model has not been configured to allow for accurate determination of the absolute value of the flux density within the material, we believe that the relative increase in field strength is physically correct, and supportive of the experimental results to be shown below.

We obtained PZT fibers (Smart Materials, Sarasota, FL) and Metglas foils (Vitrovac Inc., Hanau, German). Five pieces of PZT fibers of $180 \mu \mathrm{m}$ in thickness were oriented along their long axis to form a layer that was $10 \mathrm{~mm}$ wide and $40 \mathrm{~mm}$ long. Two interdigitated Kapton ${ }^{\circledR}$-based electrodes were then bonded to the top and bottom surfaces of the piezoelectric layer in a multi push-pull geometry. ${ }^{8}$ In order to compare the effect of Metglas foils with different lengths, Metglas foils 80 and $100 \mathrm{~mm}$ long (and $10 \mathrm{~mm}$ wide) were then laminated to both the top and bottom surfaces of the PZT composite cores.

The ME voltage coefficient $\left(\alpha_{\mathrm{ME}}\right)$ was measured as a function of dc magnetic field $\left(H_{d c}\right)$ for the different ME laminates. A $1 \mathrm{kHz}, 1$ Oe excitation was generated by driving a Helmholtz coil with a commercial lock-in amplifier (SR850). Meanwhile, a DC magnetic bias field $\left(H_{d c}\right)$ was applied along the long axis of the ME laminates using electromagnets. The strength of the DC magnetic bias was monitored using a Gauss meter. The value of $\alpha_{\mathrm{ME}}$ for ME laminates of different length exhibited similar trends with $H_{d c}$ (see Fig. 3 ). In both cases, $\alpha_{\mathrm{ME}}$ increased from roughly $0 \mathrm{~V} / \mathrm{cm}-\mathrm{Oe}$ at zero bias to a maximum value at an optimum bias condition

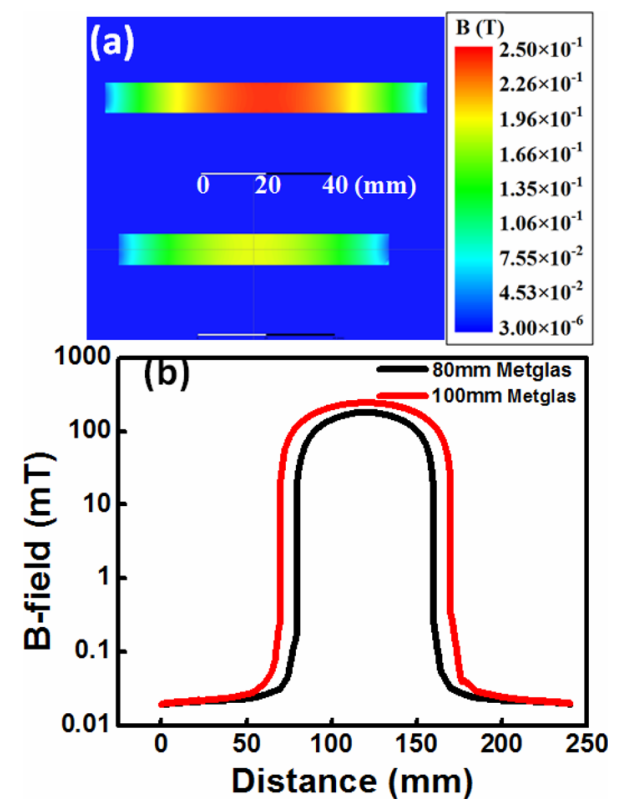

FIG. 2. (Color online) (a) In-plane magnetic field strength along the centerplane of Metglas foils in response to an arbitrarily low DC bias field, as simulated by Maxwell 3D, and (b) line scan traces of magnetic flux density along the axial centerline of Metglas foils for the $80 \mathrm{~mm}$ and $100 \mathrm{~mm}$ geometries.

and, subsequently, decreased as $H_{d c}$ increased further. The value of $\alpha_{\mathrm{ME}}$ for the laminates with the longer Metglas foils was notably higher than that for shorter ones, while also requiring smaller magnetic biases; for example, under $H_{d c}=2.5 \mathrm{Oe}$, the value of $\alpha_{\mathrm{ME}}$ was $10 \mathrm{~V} / \mathrm{cm}-O e$ for $100 \mathrm{~mm}$ long Metglas laminates, relative to $5 \mathrm{~V} / \mathrm{cm}-O e$ for $80 \mathrm{~mm}$ Metglas ones. Following the above magnetostatic modeling results, the enhancement of $\alpha_{\mathrm{ME}}$ for longer Metglas direct results from higher magnetic flux concentration.

The DC magnetic field sensitivity was then characterized for both types of sensors using an active method: ${ }^{9}$ a 100 turns coil was wrapped around the sensors which carried a small AC $(f=1 \mathrm{kHz})$ current provided by a lock-in amplifier to actively drive the ME sensors. Small changes in $H_{d c}$ achieved through $\mathrm{H}$-coil induced voltages in the PZT layer were then measured by the lock-in amplifier. In all measurements, we kept the signal-to-noise (SNR) ratio constant at 10. In our detection system, the voltage noise level was about $\mu \mathrm{V}$, thus we kept the induced voltage changes at $10 \mu \mathrm{V}$. Figures 4(a) and 4(b) show the induced output voltages from

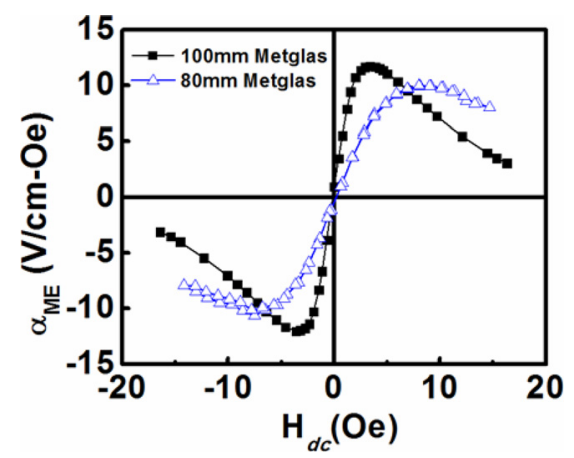

FIG. 3. (Color online) ME voltage coefficient of laminate sensor with different Metglas lengths as a function of DC bias $H_{d c}$ in response to a $1 \mathrm{Oe}, 1$ $\mathrm{kHz}$ AC magnetic flux excitation. 

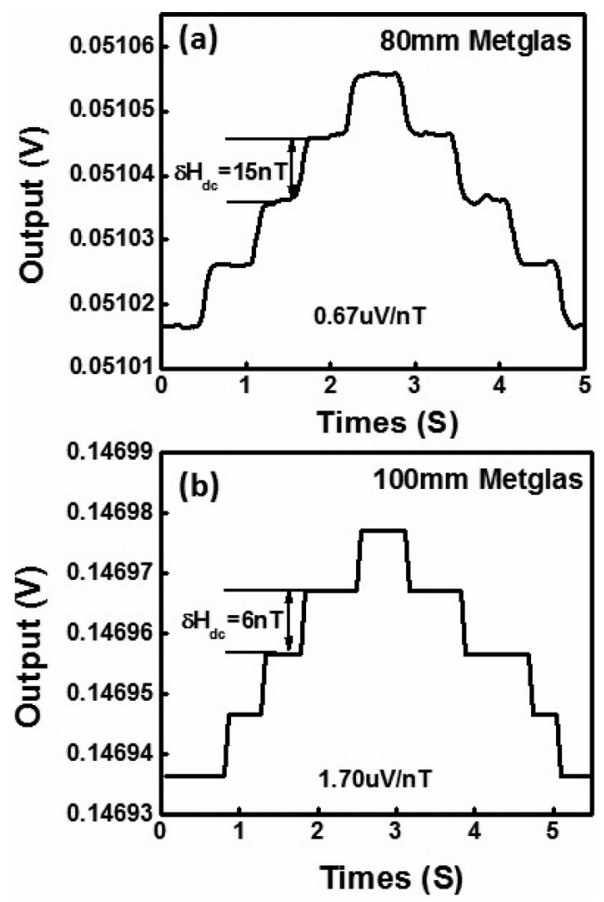

FIG. 4. Comparison of the sensitivity for Metglas/PZT laminates to small DC magnetic field changes under AC drive conditions at $f=1 \mathrm{kHz}$ and $H_{a c}=0.1$ Oe: (a) ME sensor with $8 \mathrm{~cm}$ long Metlgas and (b) ME sensor with $10 \mathrm{~cm}$ long Metglas.

the different types of Metglas/PZT laminates in response to small changes in $H_{d c}$. DC magnetic field variations as small as $H_{d c}=15 \mathrm{nT}$ could be detected under a $0.1 \mathrm{Oe}, 1 \mathrm{kHz}$ drive for the sensor with Metglas foils of $80 \mathrm{~mm}$ in length, similar to recent reports. ${ }^{10}$ However, the DC magnetic field changes as small as $6 \mathrm{nT}$ were detectable by using ME sensor with longer Metglas foils $(100 \mathrm{~mm})$; note that similar drive conditions were used. This represents a $250 \%$ increase in the DC field sensitivity.
In summary, we have developed a finite element magnetostatic model to describe the influence of Metglas length on magnetic field concentration in ME laminate sensors. We found that lengthening the Metglas layer increases the magnetic flux density over the central portion of the sensor that contains the core piezoelectric layer. A redesign of the Metglas/PZT sensor to incorporate longer Metglas foils yielded composites that displayed higher ME voltage coefficients. Although the maximum value of $\alpha_{\mathrm{ME}}$ was not enhanced by using the longer Metglas, the value of the slope $\partial\left(\alpha_{M E}\right) / \partial\left(H_{d c}\right)$ was notably enhanced over the bias range of $-2.5 \mathrm{Oe}<H_{d c}<2.5 \mathrm{Oe}$. This resulted in a $250 \%$ increase in the detection threshold (from $15 \mathrm{nT}$ to $6 \mathrm{nT}$ ), measured under a constant $\mathrm{SNR}=10$. Such high sensitivities to small DC field changes should help enable navigation based on variations in the magnitude and orientation of the local geomagnetic field.

The authors would like to acknowledge DARPA and ONR for support of this work.

${ }^{1}$ W. Cochran, H. Mouritsen, and M. Wikelski, Science 304, 405 (2004).

${ }^{2}$ K. J. Lohmann, C. M. F. Lohmann, L. M. Ehrhart, D. A. Bagley, and T. Swing, Nature(London) 428, 909 (2004).

${ }^{3}$ J. Y. Zhai, S. X. Dong, Z. P. Xing, J.-F. Li, and D. Viehland, Appl. Phys. Lett. 91, 123513 (2007).

${ }^{4}$ H. Bartehelmess, M. Halverscheid, B. Schiefenhovel, E. Heim, M. Schilling, and R. Zimmermann, IEEE Trans. Appl. Supercond. 11, 657 (2001).

${ }^{5}$ M. N. Baibich, J. M. Broto, A. Fert, F. Nguyen Van Dau, F. Petroff, P. Eitenne, G. Creuzet, A. Friederich, and J. Chazelas, Phys. Rev. Lett. 21, 2472 (1988).

${ }^{6}$ J. Y. Zhai, S. X. Dong, J.-F. Li, and D. Viehland, Appl. Phys. Lett. 88, 062510 (2006).

${ }^{7}$ C.-W. Nan, M. I. Bichurin, S. Dong, D. Viehland, and G. Srinivasan, J. Appl. Phys. 103, 031101 (2008).

${ }^{8}$ S. X. Dong, J. Y. Zhai, J.-F. Li, and D. Viehland, Appl. Phys. Lett. 89, 252904 (2006).

${ }^{9}$ S. X. Dong, J. Y. Zhai, J.-F. Li, and D. Viehland, Appl. Phys. Lett. 88, 082907 (2006).

${ }^{10}$ J. Gao, L. Shen, Y. Wang, D. Gray, J. F. Li, and D. Viehland, J. Appl. Phys. 109, 074507 (2011). 